\title{
Towards understanding the $\phi$ meson in nuclear matter with finite momentum
}

\section{Philipp Gubler*}

Advanced Science Research Center, Japan Atomic Energy Agency, Tokai, Ibaraki 319-1195,

Japan

E-mail: qublerepost.j-parc.jp

The behavior of the $\phi$ meson in nuclear matter has attracted renewed interest because of (recent and future) experiments that aim to study the $\phi$ meson properties in nuclei. Theoretically, many works have however been conducted for the $\phi$ meson at rest with respect to the nuclear medium. In these proceedings, I will review recent theoretical progress related to this topic. Non-zero momentum effects will be especially relevant for future experiments, such as E16 at J-PARC, where the $\phi$ meson will not be measured at rest, but with finite momentum with respect to the surrounding nucleus.

XIII Quark Confinement and the Hadron Spectrum - Confinement2018

31 July - 6 August 2018

Maynooth University, Ireland

${ }^{*}$ Speaker. 


\section{Introduction}

Vector mesons in dense matter have been in the focus of theoretical and experimental interest already for a long time, partly because some of them (the $\rho, \omega$ and the $\phi$ ) were predicted to be probes of the partial restoration of chiral symmetry in nuclear matter [四]. Furthermore, due to their decay into di-leptons, which do not interact strongly, they provide a relatively clear experimental signal that does not get strongly distorted by strong final state interactions and therefore in principle allow direct access to the medium to be studied.

Taking a more general point of view, understanding hadron properties from the first principles of QCD is one of the fundamental goals of hadron physics. Rapid progress in the field of lattice QCD has allowed to approach this goal in recent years, at least for the hadronic ground states in vacuum, which can now be studied at physical pion masses including dynamical quarks in state-ofthe-art lattice calculations. It is, however, still difficult even today to generalize these calculations to finite density, where lattice QCD suffers from the so-called sign problem, which prevents important sampling, which is used to numerically compute the QCD path integral in standard lattice QCD calculations, from being useful and practically feasable on a machine. To solve this problem, several approaches such as the use of the complex Langevin equation [l] , the Taylor expansion method for the small $\mu / T$ region [3], analytically continuing calculations with an imaginary chemical potential (where there is no sign problem) [团] or the use of Lefschetz thimbles [ []], have been proposed. A real solution to the sign problem and hence realistic lattice QCD simulations of hadrons at finite density however still appears to be out of reach at least in the near future.

To study the behavior of hadrons at finite density, one therefore presently needs to rely on alternative methods. These are either effective theory approaches based on hadronic degrees of freedom or methods relying on the basic degrees of freedom of $\mathrm{QCD}$, quarks and gluons. We will in these proceedings discuss recent results about the $\phi$ meson properties in nuclear matter, which were obtained using QCD sum rules in combination with the maximum entropy method [G]. For

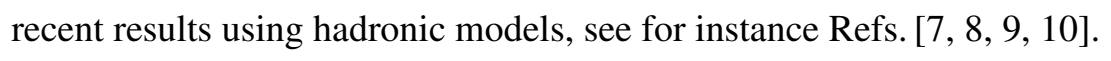

\section{The behavior of the $\phi$ meson at rest in nuclear matter}

Let us here first review a few recent theoretical results concerning the $\phi$ meson at finite density and zero momentum. As mentioned above, lattice QCD is presently not able to study this problem because of the sign problem. In QCD sum rules, which can be used to study non-zero density systems as long as the baryon density is not much above that of normal nuclear matter, one defines the follwing two-point function

$$
\Pi_{\mu v}\left(q_{0}, \boldsymbol{q}\right)=i \int d x^{4} e^{i q x}\left\langle T\left[j_{\mu}(x) j_{v}(0)\right]\right\rangle_{\rho}
$$

where to study the $\phi$ meson, we have $j_{\mu}(x)=\bar{s}(x) \gamma_{\mu} s(x)$ while $\langle\cdots\rangle_{\rho}$ is the expectation value with respect to the ground state with the baryon density fixed to $\rho$. Because of the breaking of Lorentz symmetry due to the existence of a matter rest frame, $\Pi_{\mu v}\left(q_{0}, \boldsymbol{q}\right)$ can become quite a complicated and generally involves two independent scalar components (see, for instance Refs. [ए]2, [13]). However, as long as one works in the limit of vanishing momentum, $\boldsymbol{q}=0$, which we will do 
here, it suffices to consider only the contracted component $\Pi\left(q_{0}^{2}\right)=-\frac{1}{3 q_{0}^{2}} \Pi_{\mu}^{\mu}\left(q_{0}, \boldsymbol{q}=0\right)$, which is the only independent component in this case. The analytic properties of $\Pi\left(q_{0}^{2}\right)$ allows one to derive

$$
\Pi\left(q_{0}^{2}\right)=\frac{1}{\pi} \int_{0}^{\infty} d s \frac{\operatorname{Im} \Pi(s)}{s-q_{0}^{2}-i \varepsilon},
$$

the so-called dispersion (or Kramers-Kroenig) relation. The QCD sum rule approach now makes use of the asymptotic freedom of QCD, which states that QCD becomes weakly coupled at large $\left|q_{0}^{2}\right|$. Therefore, one can compute $\Pi\left(q_{0}^{2}\right)$ in this region using perturbation theory and the operator product expansion (OPE), resulting in a power series in $1 / q_{0}^{2}$, whose coefficients contain QCD condensates of operators that appear in the OPE and parts of the respective Wilson coefficients, which are themselves computed as an expansion in $\alpha_{s}$, the strong coupling constant of QCD. $\operatorname{Im} \Pi(s)$ appearing on the right-hand side of Eq. (R.2) is nothing but the spectral function of the operator $j_{\mu}(x)$, that is, it contains all physical states with energy $\sqrt{s}$ that couple to $j_{\mu}(x)$. Note furthermore that $j_{\mu}(x)$ can be viewed as the stange part of the electromagnetic current. Therefore, $\operatorname{Im}(s)$ can also be related to the physically observable di-lepton rate. Our task would be complete if we would be able to exactly compute $\operatorname{Im} \Pi(s)$ for any density in the region of the $\phi$ meson resonance. In the vacuum, the OPE for $\Pi\left(q^{2}\right)$ gives [in the vacuum, $\Pi\left(q^{2}\right)$ depends only on $q^{2}=q_{0}^{2}-q^{2}$ due to Lorentz invariance]

$$
9 \Pi\left(q^{2}=-Q^{2}\right)=-c_{0} \log \left(\frac{Q^{2}}{\mu^{2}}\right)+\frac{c_{2}}{Q^{2}}+\frac{c_{4}}{Q^{4}}+\frac{c_{6}}{Q^{6}}+\ldots,
$$

where $\mu$ is the renormalization scale. The first few coefficients $c_{n}$ are obtained as

$$
\begin{aligned}
c_{0} & =\frac{1}{4 \pi^{2}}\left(1+\frac{\alpha_{s}}{\pi}\right), \quad c_{2}=-\frac{3 m_{s}^{2}}{2 \pi^{2}}, \\
c_{4} & =\frac{1}{12}\left\langle\frac{\alpha_{s}}{\pi} G^{2}\right\rangle_{0}+2 m_{s}\langle\bar{s} s\rangle_{0}, \\
c_{6} & =-2 \pi \alpha_{s}\left[\left\langle\left(\bar{s} \gamma_{\mu} \gamma_{5} \lambda^{a} s\right)^{2}\right\rangle_{0}+\frac{2}{9}\left\langle\left(\bar{s} \gamma_{\mu} \lambda^{a} s\right) \sum_{q=u, d, s}\left(\bar{q} \gamma_{\mu} \lambda^{a} q\right)\right\rangle_{0}\right] .
\end{aligned}
$$

Higher order terms in $\alpha_{s}$ and $m_{s}$ (strange quark mass) have been computed in the past [see Ref. [प]]

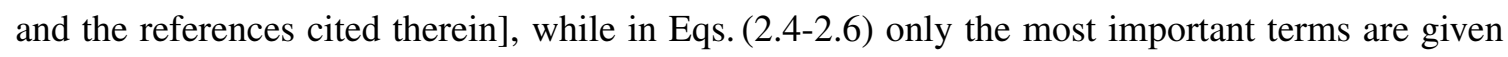
for simplicity.

To make the dispersion relation useful in preactice, it needs to be modified. As it is shown in Eq. (2.2) the integral in fact does not converge, which translates into infinite subtraction constants on the left-hand side. This issue can be averted by applying the Borel transform to $\Pi\left(q^{2}\right)$, which furthermore improves the convergence properties of the OPE. Applying the OPE to Eq. ([2.2) leads to

$$
\Pi^{\mathrm{B}}\left(M^{2}\right)=\frac{1}{M^{2}} \int_{0}^{\infty} d s e^{-s / M^{2}} \rho(s)
$$

where we have defined $\rho(s)$ as $\rho(s)=\frac{1}{\pi} \operatorname{Im} \Pi(s)$. In Ref.[W], we have applied the maximum entropy method (MEM) to analyze the sum rule of Eq. ([R.J). This technique was first developed in Ref.[目], which we refer the reader to for further details.

When going from vacuum to finite density, the OPE inputs of the sum rules are modifed. Vacuum condensates, for instance, change as a function of density. This dependence on the density 


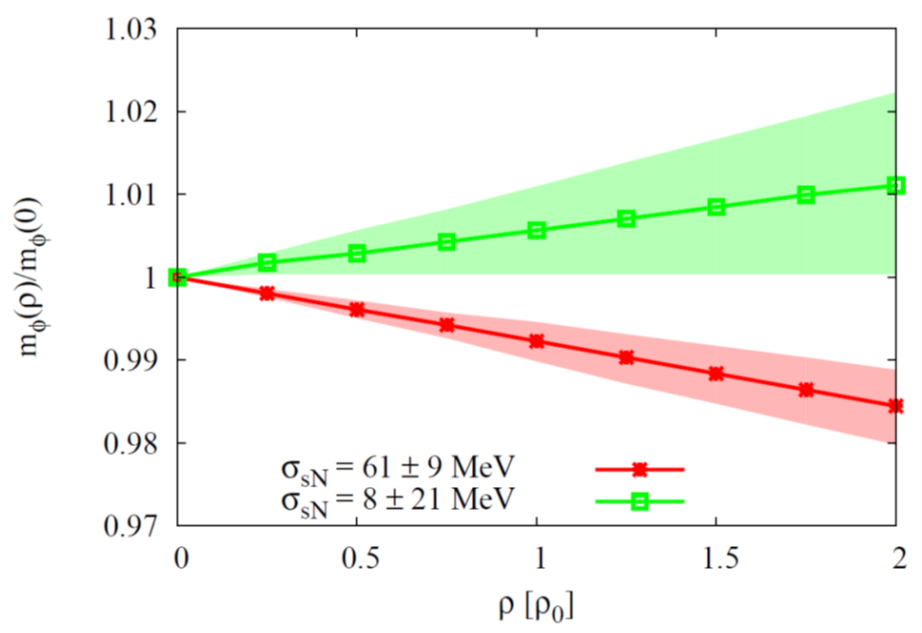

Figure 1: The $\phi$ meson mass as a function of density for two typical values of the strange sigma term $\sigma_{S N}$ obtained in relatively recent lattice QCD calculations [44, [5]] (for more recent results, see for instance, Refs. [ㅁ, [1]]). The $\phi$ meson mass is given relative to its vacuum value, while the density is given in units of the nuclear matter density $\rho_{0}$. Adapted from Ref.[ए]].

is however often not well known. At present, one can at most make statements about the value of condensates in the linear density approximation. For the condensates with lowest mass dimension, this approximation gives

$$
\begin{aligned}
\langle\bar{s} s\rangle_{\rho} & \simeq\langle\bar{s} s\rangle_{0}+\langle N|\bar{s} s| N\rangle \rho=\langle\bar{s} s\rangle_{0}+\frac{\sigma_{s N}}{m_{s}} \rho \\
\left\langle\frac{\alpha_{s}}{\pi} G^{2}\right\rangle_{\rho} & \simeq\left\langle\frac{\alpha_{s}}{\pi} G^{2}\right\rangle_{0}+\left\langle N\left|\frac{\alpha_{s}}{\pi} G^{2}\right| N\right\rangle=\left\langle\frac{\alpha_{s}}{\pi} G^{2}\right\rangle_{0}-\frac{8}{9}\left(M_{N}-\sigma_{\pi N}-\sigma_{s N}\right) \rho
\end{aligned}
$$

$M_{N}$ is the nucleon mass, $\sigma_{\pi N}$ the $\pi N$ sigma term and $\sigma_{s N}$ the nucleon strange sigma term. $\langle N|\cdots| N\rangle$ stands for the expectation value with respect to a one-nucleon state. The linear density approximation therefore completely ignores correlations between nuclei and only takes into account independent contributions of each nucleon separately. Besides the condensates that are already present in the vacuum, new non-scalar condensates can appear at finite density. For instance, a spin-2 (or twist-2) condensate is generated due to quark fields at dimension 4:

$$
\left\langle N\left|\mathscr{S} \mathscr{T} \bar{s} \gamma^{\mu} i D^{v} s\right| N\right\rangle=\frac{A_{2}^{s}}{2 M_{N}}\left(p^{\mu} p^{v}-\frac{1}{4} M_{N}^{2} g^{\mu v}\right) .
$$

$\mathscr{S} \mathscr{T}$ make thie following structure symmetric and traceless with repect to its Lorentz indices $\left[\mathscr{S} \mathscr{T} A^{\mu v} \equiv \frac{1}{2}\left(A^{\mu v}+A^{v \mu}\right)-\frac{1}{4} g^{\mu v} A_{\alpha}^{\alpha}\right]$, while $p^{\mu}$ is the nucleon four-momentum $\left(p^{2}=M_{N}^{2}\right) . A_{2}^{s}$ stands for the first moment of the strange and anti-strange quark parton distributions of the nucleon:

$$
A_{2}^{s}=2 \int_{0}^{1} d x x[s(x)+\bar{s}(x)] .
$$

More non-scalar condensates are considered for instance in Ref.[W]. We furthermore refer the interested reader to Ref.[[13], where a complete list of independent scalar and non-scalar condensates 
(up to dimension 6) that appear in the OPE of the vector correlator and their respective Wilson coefficients are given at leading order in $\alpha_{s}$. Some values of these condensates are not well known even in the linear density approximation, while others have been constrained only recently due to the emergence of new experimental data [एष]].

All the finite density effects discussed above lead to modified coefficients $c_{n}$ in Eq. 2.3. The lowest two $\left(c_{0}\right.$ and $\left.c_{2}\right)$ do not depend on density, while all others generally do. Keeping only the contributions explicitly mentioned in this section, the linear density term of $c_{4}$ (denoted as $\delta c_{4}$ here) reads

$$
\delta c_{4}=\left[\left(A_{2}^{s}-\frac{2}{27}\right) M_{N}+\frac{2}{27}\left(28 \sigma_{s N}+\sigma_{\pi N}\right)\right] \rho .
$$

For the corresponding expression for $c_{6}$, see for instance Ref.[प]]. The most dominant terms in the above expression are the $\frac{2}{27} M_{N}$ and the $\sigma_{s N}$ terms. The former originates from the modification of the gluon condensate [see Eq. (2.9)] and the latter dominantly from that of the strange quark condensate [see Eq. ([2.8)]. Especially the value of $\sigma_{s N}$, which in principle can be computed using lattice QCD, but is presently still not well constrained partly because of the numerical difficulty to compute it on the lattice with good precision. As we will demonstrate in the next paragraph, QCD sum rules relate the value of $\sigma_{s N}$ to the mass shift of the $\phi$ meson in nuclear matter.

Let us now discuss the results of the MEM analysis of the $\phi$ meson sum rules given in Ref.[W]. To keep the description simple, we in these proceedings will only show our findings on the mass shift of the $\phi$ meson peak that appears in the spectral function. Detailed estimates of the effects of broadening and other systematic uncertainties are provided in Ref.[W]. Let us just mention here that sum rules are generally not very sensitive to peak broadening effects, because these effects are eliminated to a large extent as the integral over the spectral function of Eq. ([R.T) is computed. It therefore is not very meaningful to study broadening effects using QCD sum rules and we will thus focus on the mass shift here. In Fig. $\mathbb{W}$ the $\phi$ meson mass is shown as a function of density for two representative values of $\sigma_{s N}$, obtained in fairly recent lattice QCD calculations [패, ㄷ]].

$$
\frac{m_{\phi}(\rho)}{m_{\phi}(0)}-1=\left[b_{0}-b_{1}\left(\frac{\sigma_{s N}}{1 \mathrm{MeV}}\right)\right] \frac{\rho}{\rho_{0}},
$$

It can be understood from this figure that the mass shift caused by the finite density effects depends strongly on the value of $\sigma_{s N}$. This dependence becomes more evident in Fig. [2, where the $\phi$ meson mass at normal nuclear matter density $\rho_{0}$ is shown as a function of $\sigma_{s N}$. It is observed in this plot that the $\phi$ meson mass shift at $\rho_{0}$ depends linearly on $\sigma_{s N}$ and is positive for $\sigma_{s N}<35 \mathrm{MeV}$, and turns negative for $\sigma_{s N}>35 \mathrm{MeV}$. To cast this result in a simple formula, we have fitted the $\phi$ meson mass shift at $\rho_{0}$ by a constant plus a term linear in $\sigma_{s N}$, for which we get $b_{0}=(1.00 \pm 0.34) \times 10^{-2}$ and $b_{1}=(2.86 \pm 0.48) \times 10^{-4}$, meaning that the mass shift switches its sign at a $\sigma_{s N} / 1 \mathrm{MeV}$ value of $b_{0} / b_{1}=34.9 \pm 13.1$.

\section{Towards studying the $\phi$ meson in nuclear matter with non-zero momentum}

So far, we have only studied the $\phi$ meson at rest with respect to nulear matter, primarily because this case is most straghtforward to compute. From an experimental point of view, however, 


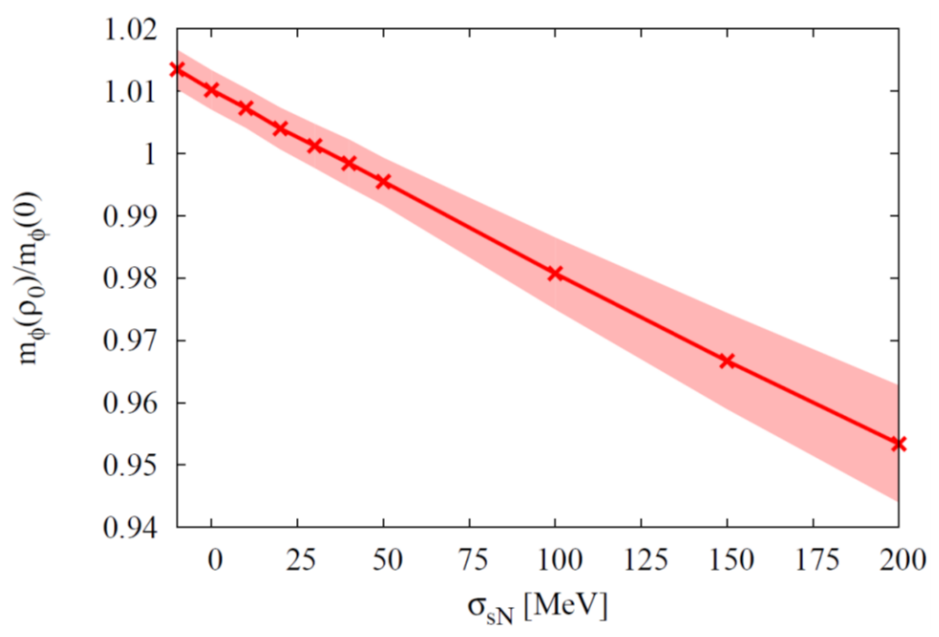

Figure 2: The $\phi$ meson mass at normal nuclear matter density $\rho_{0}$ as a function of $\sigma_{s N}$. Adapted from Ref.[ए]].

this is not very realistic, as the $\phi$ will very likely move with some non-vanishing velocity once it is produced inside the nucleus through some kind of reaction. Therefore, the effect of such nonzero velocity has to be studied. Long time ago, this was already done in Ref. [10], using the OPE results that were known at that time. Recently, the OPE of the vector channel was completed up to operators with mass dimension 6 , including non-scalar gluonic dimension 6 operators, whose Wilson coefficients have not been obtained before [피]. With these results at hand, we can now study the effect of non-zero velocity/momentum with much less theoretical uncertainty. Calculations towards this direction are presently ongoing and we hope to obtain meaningful results soon. Such results will be especially meaningful for the future E16 experiment at J-PARC, for which on propagated goal is to measure the mass shift of the $\phi$ at normal nuclear matter density as a function of momentum [R2] $]$.

\section{Summary and Conclusions}

In these proceedings, we have discussed the $\phi$ meson mass shift in nuclear matter and its potential to constrain strangeness sigma term $\sigma_{S N}$. An experimental measurement of this mass shift and direct lattice QCD computations of $\sigma_{s N}$ will help to obtain precise information about the way chiral symmetry in the strange sector is restored in a medium with non-zero baryon density. Furthermore, we have mentioned the possibily to take finite momentum effects into account in future calculations. Such calculations will hopefully provide useful guidance for the E16 experiment at J-PARC, which is scheduled to start in early 2020.

\section{Acknowledgments}

The author thanks HyungJoo Kim, Su Houng Lee, Keisuke Ohtani, Makoto Oka, Kie Sang Jeong and Wolfram Weise for the fruitful collaborations that are mentioned in these proceedings. 


\section{References}

[1] T. Hatsuda and S. H. Lee, Phys. Rev. C 46, no. 1, R34 (1992).

[2] G. Aarts, E. Seiler and I. O. Stamatescu, Phys. Rev. D 81, 054508 (2010) [arXiv:0912.3360 [hep-lat]].

[3] C. R. Allton, S. Ejiri, S. J. Hands, O. Kaczmarek, F. Karsch, E. Laermann, C. Schmidt and L. Scorzato, Phys. Rev. D 66, 074507 (2002) [hep-lat/0204010].

[4] M. G. Alford, A. Kapustin and F. Wilczek, Phys. Rev. D 59, 054502 (1999) [hep-lat/9807039].

[5] M. Cristoforetti et al. [AuroraScience Collaboration], Phys. Rev. D 86, 074506 (2012) [arXiv:1205.3996 [hep-lat]].

[6] P. Gubler and M. Oka, Prog. Theor. Phys. 124, 995 (2010) [arXiv:1005.2459 [hep-ph]].

[7] P. Gubler and W. Weise, Phys. Lett. B 751, 396 (2015) [arXiv:1507.03769 [hep-ph]].

[8] P. Gubler and W. Weise, Nucl. Phys. A 954, 125 (2016) [arXiv:1602.09126 [hep-ph]].

[9] D. Cabrera, A. N. Hiller Blin and M. J. Vicente Vacas, Phys. Rev. C 95, no. 1, 015201 (2017) [arXiv:1609.03880 [nucl-th]].

[10] J. J. Cobos-Martinez, K. Tsushima, G. Krein and A. W. Thomas, Phys. Lett. B 771, 113 (2017) [arXiv:1703.05367 [nucl-th]].

[11] P. Gubler and K. Ohtani, Phys. Rev. D 90, no. 9, 094002 (2014) [arXiv:1404.7701 [hep-ph]].

[12] S. s. Kim and S. H. Lee, Nucl. Phys. A 679, 517 (2001) [nucl-th/0002002].

[13] H. Kim, P. Gubler and S. H. Lee, Phys. Lett. B 772, 194 (2017) Erratum: [Phys. Lett. B 779, 498 (2018)] [arXiv:1703.04848 [hep-ph]].

[14] W. Freeman et al. [MILC Collaboration], Phys. Rev. D 88, 054503 (2013) [arXiv:1204.3866 [hep-lat]].

[15] H. Ohki et al. [JLQCD Collaboration], Phys. Rev. D 87, 034509 (2013) [arXiv:1208.4185 [hep-lat]].

[16] S. Durr et al., Phys. Rev. Lett. 116, no. 17, 172001 (2016) [arXiv:1510.08013 [hep-lat]].

[17] N. Yamanaka et al. [JLQCD Collaboration], Phys. Rev. D 98, no. 5, 054516 (2018) [arXiv:1805.10507 [hep-lat]].

[18] P. Gubler, K. S. Jeong and S. H. Lee, Phys. Rev. D 92, no. 1, 014010 (2015) [arXiv:1503.07996 [hep-ph]].

[19] S. H. Lee, Phys. Rev. C 57, 927 (1998) Erratum: [Phys. Rev. C 58, 3771 (1998)] [nucl-th/9705048].

[20] K. Aoki [J-PARC E16 Collaboration], arXiv:1502.00703 [nucl-ex]. 